\title{
Editorial
}

\section{Suicide Prevention in Primary Care}

\section{The Opportunity for Intervention}

\author{
Faraz Mughal1,2,3, Hayley C. Gorton ${ }^{4}$, Maria Michail ${ }^{5}$, \\ Jo Robinson ${ }^{6,7}$, and Pooja Saini ${ }^{8}$
}
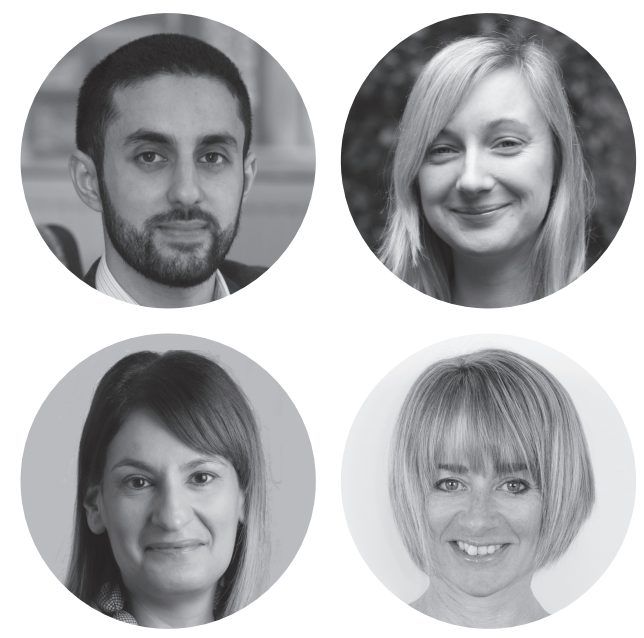

'School of Medicine, Keele University, Staffordshire, UK

${ }_{2}^{2}$ NIHR Greater Manchester Patient Safety Translational Research Centre,

The University of Manchester, UK

${ }^{3}$ Unit of Academic Primary Care, University of Warwick, Coventry, UK

${ }^{4}$ School of Applied Sciences, University of Huddersfield, UK

${ }^{5}$ Institute for Mental Health, School of Psychology, University of Birmingham, UK

${ }^{6}$ Orygen, Parkville, VIC, Australia

${ }^{7}$ Centre for Youth Mental Health, The University of Melbourne, Parkville, VIC, Australia

${ }^{8}$ Faculty of Health, School of Psychology, Liverpool John Moores University, UK

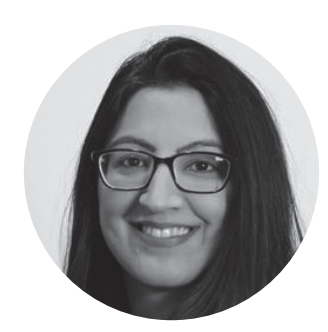

Suicide is a leading cause of death internationally, and self-harm significantly increases the risk of suicide (Hawton et al., 2015; World Health Organization, 2014). To tackle this global health crisis a multisystem, inter-faceted, joined-up approach across health and social care, education, judicial, financial, and voluntary sectors is needed. Within this, the health setting of primary care holds unlocked potential for suicide prevention (Michail et al., 2020). In this editorial, we outline the importance of focusing on primary care for suicide prevention and introduce a new special interest group for the International Association for Suicide Prevention.

\section{What Is Primary and Community Health Care?}

The World Health Organization defines primary healthcare as a whole-society approach to health and well-being centered on the preferences and needs of individuals, families, and communities, which focuses on the interrelated aspects of physical, mental, and social health and wellbeing (World Health Organization \& UNICEF, 2018). This approach needs to encompass the continuum of healthcare - from health promotion and prevention, to treatment and rehabilitation - within people's daily environments (World Health Organization \& UNICEF, 2018).

Primary care services often provide a first point of contact in a health system and generally include the services of general practice, community pharmacy, as well as dental and optometry teams, although this may differ across lower- and middle-income countries (LMICs; Charles, 2019). Community health services strive to support people of any age and often with complex multiple problems in their homes and communities. They closely complement primary care services and cover a diverse range of activities that can be both difficult to define and can vary across regions and countries (Charles, 2019). In the National Health Service (NHS) of the United Kingdom (UK), examples of community health services include community occupational therapy and physiotherapy, health visiting and sexual health services, nursing homes, school nursing, the voluntary sector, and caring services (Charles, 2019).

\section{Why Primary Care for Suicide Prevention?}

Robust evidence has identified increasing rates of selfharm in young and older people recorded in general practice electronic patient health records (Marchant et al., 
2019; Morgan et al., 2018; Morgan et al., 2017). This indicates an increased workload for primary care teams in relation to self-harm but highlights the opportunity for early identification and intervention. Carr et al. (2016) found that only a small number of patients recorded in primary care to have harmed themselves were referred for specialist support, thus identifying the need to develop support and interventions within primary care.

A recent systematic review found that general practitioners (GPs) and family medicine physicians have an important role in self-harm management, which encompasses frontline assessment and treatment, ongoing management in primary care, and referral to specialist care (Mughal et al., 2020). Patients have stated that screening for suicidal thoughts should occur in primary care and GPs feel similarly about patients with depression (Bajaj et al., 2008).

The Programme for Improving Mental Health Care (PRIME) consortium found that across Ethiopia, Uganda, South Africa, India, and Nepal, $10 \%$ of people presenting to primary care facilities reported suicidal thoughts in the past year (Jordans et al., 2018). The PRIME consortium also found that having a mental health problem, being female, and younger in age were all features associated with suicidal behavior (Jordans et al., 2018). These findings from LMICs stress the importance of engaging in suicide prevention in primary care and the need for specific strategies to reduce suicide in these settings. This also represents an opportunity for tailored screening to help primary care professionals identify suicidal thoughts, intent, and possible future risk of suicide early, to enable intervention.

A qualitative study of primary healthcare professionals in Nicaragua found that primary healthcare staff wanted to improve their ability to identify and treat young people with suicidal behavior and saw primary care as an important setting in which to do this, albeit within the constraints of limited time and resources (Obando Medina et al., 2014).

A recent study found that $85 \%$ of people who died by suicide in Wales were in recent contact with general practice; and that patients most often consulted for mental health concerns (John et al., 2020). An English report found that risk of suicide increased with more GP consultations especially in the 3 months before suicide (National Confidential Inquiry Into Suicide and Homicide by People With Mental Illness [NCISH], 2014). This highlights the importance of making each healthcare contact count in primary care and aiming for enhanced mental health care of all patients (John et al., 2020; Pearson et al., 2009).

Community pharmacy staff recognize they are an underused yet key component of a system response to suicide prevention (Gorton et al., 2021; Gorton et al., 2019). The value of this has been recognized in England through the incentivization of community pharmacy staff to complete suicide prevention training before February 2021 (Pharmaceutical Services Negotiating Committee, n.d.): We are not aware of similar schemes in other countries. However, since 2018, all pharmacists registered in Washington state in the United States have been mandated by law to undertake suicide prevention training (Graves et al., 2018).

In the recent NHS Long-Term Plan (National Health Service, 2019), there is a commitment to the development of integrated and enhanced primary care and community mental health services in the UK. As primary and community health services develop and evolve over time, it is paramount that both healthcare settings in countries become more connected. This will provide a strong foundation for the assessment and management of people with selfharm and suicidal behaviors internationally (Michail et al., 2020; Senarathna et al., 2008).

\section{Primary Care Interventions}

There have been a number of studies that have evaluated primary-care-delivered interventions for suicide prevention (Milner et al., 2017). Almeida et al. (2012) conducted a cluster randomized controlled trial (RCT) of a GPtargeted educational intervention (personalized feedback and educational material) for older adults in Australia and found that the intervention led to a significant reduction in self-harm behavior during follow-up. A four-level intervention program aimed at improving outcomes for patients with depression in Nuremberg, Germany, included the training of primary care physicians across 12 sessions, provision of educational videos, and the availability of a specialist hotline for individual patient advice, and resulted in a significant reduction in suicide attempts compared with the control region (Hegerl et al., 2006).

A collaborative care intervention of trained care managers supporting primary care physicians in the management of older adults with depression resulted in good adherence to depression medication and reduced suicidal ideation over 2 years (Alexopoulos et al., 2009). In addition, a training program across Germany, Hungary, Ireland, and Portugal found that training community facilitators such as social workers, nurses, and teachers can improve facilitator knowledge and confidence in suicidal behaviors, thus encouraging early detection and intervention (Coppens et al., 2014).

In Iran, an integrated tiered suicide prevention program into primary healthcare resulted in higher detection of depressive disorders and lower numbers of suicides (Malakouti et al., 2015). However, well-conducted RCTs such as those by Grimholt et al. (2015; structured continued GP follow-up for adults who self-poisoned) and Bennewith 
et al. (2002; an invitation for a GP consultation and clinical guidelines for the GP after hospital presenting selfharm) resulted in no significant reductions in self-harm at follow-up.

The studies that offer promising findings need adaption and replication across countries; and the development and testing of new complex and system level interventions are important in understanding how primary care can effectively intervene. These must be undertaken with parallel process evaluation to gain insights into what components work for who, and why, and also in understanding what patients and clinicians find acceptable.

The development and testing of new interventions such as the Community Outpatient Psychotherapy Engagement Service for Self-harm (COPESS), and an electronic clinical decision support tool for the management of suicidality in primary care, may soon yield positive results (Horrocks et al., 2018; Saini et al., 2021).

\section{Coronavirus Implications}

The coronavirus disease (COVID-19) pandemic is refocusing attention to the importance of mental health in individuals, communities, and nations and to the risk of suicide that COVID-19 brings (Gunnell et al., 2020). Recent evidence identified that suicidal thoughts especially in young adults and those from socially disadvantaged backgrounds rose during the COVID-19 pandemic (O'Connor et al., 2020); and older adults with multimorbidity in Hong Kong reported significantly higher levels of isolation and anxiety early in the COVID-19 outbreak (Wong et al., 2020).

At present, the impact of COVID-19 on rates of suicide internationally is largely unchanged, and statistical analyses found evidence of a reduction in suicide rates early in the pandemic compared with pre-pandemic expected levels across 12 countries or areas (Pirkis et al., 2021). However, there is a need for continual monitoring of rates and to remain vigilant moving forward into the pandemic.

It is necessary to monitor the pandemic and post-pandemic periods within and across countries where economic and social hardships may persist in the pandemic recovery period while vaccination programs are established. The formation of the international research collaboration for suicide prevention in the context of COVID-19 is welcome (Niederkrotenthaler et al., 2020), but it is crucial that both primary care and community settings are focuses of new and ongoing COVID-19-related research (Mughal et al., 2021).

\section{Special Interest Group}

A new international collaborative, the Suicide Prevention in Primary Care Special Interest Group (SIG) of the International Association of Suicide Prevention, has been established. This international collaborative network will bring together clinicians, researchers, policymakers, commissioners, and people with lived experience. It will identify future research priorities and build the evidence base in both developing and developed countries. The new knowledge generated will be used to harness the potential of, and to identify, opportunities to optimize primary care and community settings in a system-wide approach to suicide prevention across the globe.

\section{Future Needs}

There have been recent strides in building the evidence base of suicide prevention in primary care (Almeida et al., 2012; Bellairs-Walsh et al., 2020; Bennewith et al., 2002; Beškovnik et al., 2011; Farr et al., 2021; Hegerl et al., 2006; Milner et al., 2017; Morgan et al., 2017; Rihmer et al., 2012; Saini et al., 2021; Saini et al., 2010); however, the field remains under-developed and under-researched.

With primary care settings often being the first point of contact in most countries, it is imperative that new ways of identifying and intervening for people at risk of suicide are found, grounded in high-quality research, and informed by patient and public involvement. The new suicide prevention in primary care SIG will support this.

Future research needs to focus not only on interventions at the population level, but also on interventions at an individual level, that are inclusive and personalized, with an understanding of what works for whom, why, and when. There are opportunities to improve clinical and community practice, informed by education and training, but this has to be evidence-informed and continually evaluated to ensure resources are best utilized.

Primary and community healthcare settings have been somewhat neglected over the years in preference of specialist mental health settings in suicide prevention, but they offer an important opportunity for early identification, intervention, and ongoing support for people who experience suicidal behavior. Primary and community care has important potential that needs further exploration through robust research that considers implementation and sustainability early on, and that harnesses the expertise of patients and the public. This will culminate in whole-person care for patients, families, and societies, in the global challenge and ambition of preventing suicide around the world. 


\section{References}

Alexopoulos, G. S., Reynolds, C. F., 3rd, Bruce, M. L., Katz, I. R., Raue, P. J., Mulsant, B. H., Oslin, D. W., \& Ten Have, T. (2009). Reducing suicidal ideation and depression in older primary care patients: 24-month outcomes of the PROSPECT study. Am $J$ Psychiatry, 166(8), 882-890. https://doi.org/10.1176/appi. ajp.2009.08121779

Almeida, O. P., Pirkis, J., Kerse, N., Sim, M., Flicker, L., Snowdon, J., Draper, B., Byrne, G., Goldney, R., Lautenschlager, N. T., Stocks, N., Alfonso, H., \& Pfaff, J. J. (2012). A randomized trial to reduce the prevalence of depression and self-harm behavior in older primary care patients. Annals of Family Medicine, 10(4), 347356. https://doi.org/10.1370/afm.1368

Bajaj, P., Borreani, E., Ghosh, P., Methuen, C., Patel, M., \& Joseph, M. (2008). Screening for suicidal thoughts in primary care: The views of patients and general practitioners. Mental Health in Family Medicine, 5(4), 229-235.

Bellairs-Walsh, I., Perry, Y., Krysinska, K., Byrne, S. J., Boland, A., Michail, M., Lamblin, M., Gibson, K. L., Lin, A., Li, T. Y., Hetrick, S., \& Robinson, J. (2020). Best practice when working with suicidal behaviour and self-harm in primary care: A qualitative exploration of young people's perspectives. Bmj Open, 10(10), e038855. https://doi.org/10.1136/bmjopen-2020-038855

Bennewith, O., Stocks, N., Gunnell, D., Peters, T. J., Evans, M. O., \& Sharp, D. J. (2002). General practice based intervention to prevent repeat episodes of deliberate self harm:Cluster randomised controlled trial. BMJ: British Medical Journal, 324(7348), 12541254. https://doi.org/10.1136\%2Fbmj.324.7348.1254

Beškovnik, L., Juričič, N. K., \& Svab, V. (2011). Suicide index reduction in Slovenia: The impact of primary care provision. Mental Health in Family Medicine, 8(1), 51-55.

Carr, M. J., Ashcroft, D. M., Kontopantelis, E., Awenat, Y., Cooper, J., Chew-Graham, C., Kapur, N., \& Webb, R. T. (2016). The epidemiology of self-harm in a UK-wide primary care patient cohort, 2001-2013. BMC Psychiatry, 16(1), 53. https://doi.org/10.1186/ s12888-016-0753-5

Charles, A. (2019). Community health services explained. https:// www.kingsfund.org.uk/publications/community-health-ser vices-explained

Coppens, E., Van Audenhove, C., Iddi, S., Arensman, E., Gottlebe, K., Koburger, N., Coffey, C., Gusmão, R., Quintão, S., Costa, S., Székely, A., \& Hegerl, U. (2014). Effectiveness of community facilitator training in improving knowledge, attitudes, and confidence in relation to depression and suicidal behavior: results of the OSPI-Europe intervention in four European countries. Journal of Affective Disorders, 165, 142-150. https://doi.org/10.1016/j. jad.2014.04.052

Farr, J., Surtees, A. D. R., Richardson, H., \& Michail, M. (2021). Exploring the processes involved in seeking help from a general practitioner for young people who have been at risk of suicide. International Journal of Environmental Research and Public Health, 18(4). https://doi.org/10.3390/ijerph18042120

Gorton, H., O'Reilly, C., Berry, H., Gardner, D., \& Murphy, A. (2021). Understanding pharmacy staff attitudes and experience relating to suicide. In Z. Babar (Ed.), Pharmacy practice research case studies (pp. 111-132). Elsevier Inc.

Gorton, H. C., Littlewood, D., Lotfallah, C., Spreadbury, M., Wong, K. L., Gooding, P., \& Ashcroft, D. M. (2019). Current and potential contributions of community pharmacy teams to self-harm and suicide prevention: A qualitative interview study. Plos One, 14(9), e0222132. https://doi.org/10.1371/journal.pone.0222132

Graves, J. M., Mackelprang, J. L., Van Natta, S. E., \& Holliday, C. (2018). Suicide prevention training: Policies for health care professionals across the United States as of October 2017.
American Journal of Public Health, 108(6), 760-768. https://doi. org/10.2105/ajph.2018.304373

Grimholt, T. K., Jacobsen, D., Haavet, O. R., Sandvik, L., Jorgensen, T., Norheim, A. B., \& Ekeberg, O. (2015). Effect of systematic follow-up by general practitioners after deliberate self-poisoning: A randomised controlled trial. PLoS One, 10(12), e0143934 https://doi.org/10.1371/journal.pone.0143934

Gunnell, D., Appleby, L., Arensman, E., Hawton, K., John, A., Kapur N., Khan, M., O'Connor, R. C., \& Pirkis, J. (2020). Suicide risk and prevention during the COVID-19 pandemic. Lancet Psychiatry, 7(6), 468-471. https://doi.org/10.1016/s2215-0366(20)30171-1

Hawton, K., Bergen, H., Cooper, J., Turnbull, P., Waters, K., Ness, J., \& Kapur, N. (2015). Suicide following self-harm: Findings from the multicentre study of self-harm in England, 2000-2012. Journal of Affective Disorders, 175, 147-151. https://doi.org/10.1016/j. jad.2014.12.062

Hegerl, U., Althaus, D., Schmidtke, A., \& Niklewski, G. (2006). The alliance against depression: 2-year evaluation of a community-based intervention to reduce suicidality. Psychological Medicine, 36(9), 1225-1233. https://doi.org/10.1017/s00 3329170600780x

Horrocks, M., Michail, M., Aubeeluck, A., Wright, N., \& Morriss, R. (2018). An electronic clinical decision support system for the assessment and management of suicidality in primary care: Protocol for a mixed-methods study. JMIR Research Protocols, 7(12), e11135. https://doi.org/10.2196/11135

John, A., DelPozo-Banos, M., Gunnell, D., Dennis, M., Scourfield, J., Ford, D., Kapur, N., \& Lloyd, K. (2020). Contacts with primary and secondary healthcare prior to suicide: case-control whole-population-based study using person-level linked routine data in Wales, UK, 2000-2017. The British Journal of Psychiatry, 127(6), 717-724. https://doi.org/10.1192/bjp.2020.137

Jordans, M., Rathod, S., Fekadu, A., Medhin, G., Kigozi, F., Kohrt, B., Luitel, N., Petersen, I., Shidhaye, R., \& Ssebunnya, J. (2018). Suicidal ideation and behaviour among community and health care seeking populations in five low-and middle-income countries: A cross-sectional study. Epidemiology and Psychiatric Sciences, 27(4), 393-402. https://doi.org/10.1017/ s2045796017000038

Malakouti, S. K., Nojomi, M., Poshtmashadi, M., Hakim Shooshtari, M., Mansouri Moghadam, F., Rahimi-Movaghar, A., Afghah, S., Bolhari, J., \& Bazargan-Hejazi, S. (2015). Integrating a suicide prevention program into the primary health care network: A field trial study in Iran. BioMed Research International, 2015, 193729. https://doi.org/10.1155/2015/193729

Marchant, A., Turner, S., Balbuena, L., Peters, E., Williams, D., Lloyd, K., Lyons, R., \& John, A. (2019). Self-harm presentation across healthcare settings by sex in young people: an e-cohort study using routinely collected linked healthcare data in Wales, UK. Archives of Disease in Childhood, 105(4), 347-354. https://doi. org/10.1136/archdischild-2019-317248

Michail, M., Mughal, F., \& Robinson, J. (2020). Suicide prevention in young people: Optimising primary care. British Journal of General Practice, 70(692), 104-105. https://doi.org/10.3399/ bjgp20X708329

Milner, A., Witt, K., Pirkis, J., Hetrick, S., Robinson, J., Currier, D., Spittal, M. J., Page, A., \& Carter, G. L. (2017). The effectiveness of suicide prevention delivered by GPs: A systematic review and meta-analysis. Journal of Affective Disorders, 210, 294-302. https://doi.org/10.1016/j.jad.2016.12.035

Morgan, C., Webb, R. T., Carr, M. J., Kontopantelis, E., Chew-Graham, C. A., Kapur, N., \& Ashcroft, D. M. (2018). Self-harm in a primary care cohort of older people: Incidence, clinical management, and risk of suicide and other causes of death. Lancet Psychiatry, 5(11), 905-912. https://doi.org/10.1016/s22150366(18)30348-1 
Morgan, C., Webb, R. T., Carr, M. J., Kontopantelis, E., Green, J., Chew-Graham, C. A., Kapur, N., \& Ashcroft, D. M. (2017). Incidence, clinical management, and mortality risk following self harm among children and adolescents: Cohort study in primary care. BMJ, 359, j4351. https://doi.org/10.1136/bmj.j4351

Mughal, F., House, A., Kapur, N., Webb, R. T., \& Chew-Graham, C. A. (2021). Suicide prevention and COVID-19: The role of primary care during the pandemic and beyond. British Journal of General Practice, 71(706), 200-201. https://doi.org/10.3399/bjgp 21X715637

Mughal, F., Troya, M. I., Dikomitis, L., Chew-Graham, C. A., Corp, N., \& Babatunde, O. O. (2020). Role of the GP in the management of patients with self-harm behaviour: A systematic review. British Journal of General Practice, 70(694), e364-e737. https://doi. org/10.3399/bjgp20X708257

National Confidential Inquiry Into Suicide and Homicide by People With Mental Illness (NCISH). (2014). Suicide in primary care in England: 2002-2011. University of Manchester. https://docu ments. manchester.ac.uk/display.aspx?DoclD=37574

National Health Service. (2019). The NHS long term plan. https:// www.longtermplan.nhs.uk/wp-content/uploads/2019/08/nhslong-term-plan-version-1.2.pdf

Niederkrotenthaler, T., Gunnell, D., Arensman, E., Pirkis, J., Appleby, L., Hawton, K., John, A., Kapur, N., Khan, M., O'Connor, R. C., \& Platt, S. (2020). Suicide research, prevention, and COVID-19. Crisis, 41(5), 321-330. https://doi.org/10.1027/0227-5910/ a000731

O'Connor, R. C., Wetherall, K., Cleare, S., McClelland, H., Melson, A. J., Niedzwiedz, C. L., O'Carroll, R. E., O'Connor, D. B., Platt, S., Scowcroft, E., Watson, B., Zortea, T., Ferguson, E., \& Robb, K. A. (2020). Mental health and wellbeing during the COVID-19 pandemic: Longitudinal analyses of adults in the UK COVID-19 Mental Health \& Wellbeing study. The British Journal of Psychiatry. Advance online publication. https://doi.org/10.1192/ bjp.2020.212

Obando Medina, C., Kullgren, G., \& Dahlblom, K. (2014). A qualitative study on primary health care professionals' perceptions of mental health, suicidal problems and help-seeking among young people in Nicaragua. BMC Family Practice, 15(1), 129. https://doi.org/10.1186/1471-2296-15-129

Pearson, A., Saini, P., Da Cruz, D., Miles, C., While, D., Swinson, N., Williams, A., Shaw, J., Appleby, L., \& Kapur, N. (2009). Primary care contact prior to suicide in individuals with mental illness. British Journal of General Practice, 59(568), 825-832. https:// doi.org/10.3399/bjgp09X472881

Pharmaceutical Services Negotiating Committee. (n.d.). PQS 2020/21 Part 2. https://psnc.org.uk/services-commissioning/ pharmacy-quality-scheme/pqs-2020-21-part-2/

Pirkis, J., John, A., Shin, S., DelPozo-Banos, M., Arya, V., Analuisa-Aguilar, P., Appleby, L., Arensman, E., Bantjes, J., Baran, A., Bertolote, J. M., Borges, G., Brečić, P., Caine, E., Castelpietra, G., Chang, S. S., Colchester, D., Crompton, D., Curkovic, M., ... Spittal, M. J. (2021). Suicide trends in the early months of the COVID-19 pandemic: An interrupted time-series analysis of preliminary data from 21 countries. Lancet Psychiatry. https:// doi.org/10.1016/s2215-0366(21)00091-2

Rihmer, Z., Gonda, X., Eôry, A., Kalabay, L., \& Torzsa, P. (2012). Screening of depression in primary care in Hungary and its importance in suicide prevention. Psychiatria Hungarica: A Magyar Pszichiatriai Tarsasag tudomanyos folyoirata, 27(4), 224-232.

Saini, P., Hunt, A., Taylor, P., Mills, C., Clements, C., Mulholland, H., Kullu, C., Hann, M., Duarte, R., Mattocks, F., \& Guthrie, E. (2021). Community Outpatient Psychotherapy Engagement Service for Self-Harm (COPESS):A feasibility trial protocol. ResearchSquare (preprint). https://doi.org/10.21203/rs.3.rs-264005/v1

Saini, P., Windfuhr, K., Pearson, A., Da Cruz, D., Miles, C., Cordingley, L., While, D., Swinson, N., Williams, A., Shaw, J., Appleby, L.,
\& Kapur, N. (2010). Suicide prevention in primary care: General practitioners' views on service availability. BMC Research Notes, 3, 246. https://doi.org/10.1186/1756-0500-3-246

Senarathna, L., Adams, J., De Silva, D., Buckley, N. A., \& Dawson, A. H. (2008). Personal and professional challenges in the management of deliberate self-poisoning patients in rural Sri Lanka: A qualitative study of rural hospital doctors' experiences and perceptions. BMC Public Health, 8(1), 373. https://doi. org/10.1186/1471-2458-8-373

Wong, S. Y. S., Zhang, D., Sit, R. W. S., Yip, B. H. K., Chung, R. Y., Wong, C. K. M., Chan, D. C. C., Sun, W., Kwok, K. O., \& Mercer, S. W. (2020). Impact of COVID-19 on loneliness, mental health, and health service utilisation: A prospective cohort study of older adults with multimorbidity in primary care. British Journal of General Practice, 70(700), e817-e824. https://doi.org/10.3399/bjg p20X713021

World Health Organization. (2014). Preventing suicide: A global imperative. https://www.who.int/mental_health/suicide-prevention/world_report_2014/en/

World Health Organization \& UNICEF. (2018). A vision for primary health care in the 21st century. https://apps.who.int/iris/bitstream/handle/10665/328065/WHO-HIS-SDS-2018.15-eng. pdf?sequence $=1 \&$ isAllowed $=y$

Published online June 29, 2021

\section{Conflict of Interest}

Faruz Mughal is a member of the current National Institute for Health and Care Excellence self-harm clinical guideline development committee.

The other authors have no conflicts of interests to declare.

\section{Funding}

This article received no funding.

FM is funded by a NIHR Doctoral Fellowship (NIHR300957).

PS is chief investigator of the NIHR Research for Patient Benefit funded COPESS study (NIHR200543). The views in this paper are those of the authors, and not necessarily those of the NHS, NIHR, or Departmentof Health and Social Care.

JR is supported by a National Health and Medical Research Council Career Development Fellowship (1142348).

\section{Faraz Mughal}

School of Medicine

Keele University

Staffordshire

UK

f.mughal@keele.ac.uk

Dr. Faraz Mughal is a general practitioner and National Institute for Health Research (NIHR) doctoral fellow at the School of Medicine, Keele University, UK. His research focuses on improving suicide prevention in primary care using evidence syntheses, qualitative methods, and complex intervention development and evaluation, all informed by patient and public involvement.

Hayley C. Gorton is a senior lecturer in pharmacy practice at the University of Huddersfield in the UK, and a pharmacist. Her research includes big data epidemiology related to suicide and selfharm, and the role of community pharmacy teams in suicide prevention. She is a fellow of the Winston Churchill Memorial Trust. 
Dr. Michail is a senior research fellow at the Institute for Mental Health, University of Birmingham, where she leads the suicide and self-harm research group. Dr. Michail has been awarded a Marie Skłodowska-Curie Global Fellowship to explore how systems modelling can inform strategic decision making for suicide prevention in young people.

Jo Robinson is an associate professor at Orygen, where she leads the suicide prevention research unit. Her work focuses on developing and testing novel interventions that specifically target at risk youth across settings and on the translation of research evidence into practice and policy. She is also a vice president of the International Association of Suicide Prevention (IASP).

Dr. Pooja Saini is a chartered psychologist and reader in suicide and self-harm prevention at Liverpool John Moores University (LJMU). Dr. Saini is well published and leads the LJMU suicide and self-harm research group. Her work includes public engagement, knowledge exchange, implementation science, and expertise in quantitative and qualitative research methods. 